\title{
The chloride horse and normal saline cart: the association of crystalloid choice with acid base status and patient outcomes in kidney transplant recipients
}

\author{
Stuart A. McCluskey, MD, PhD (1) · Justyna Bartoszko, MD
}

Received: 6 December 2019/Revised: 6 December 2019/Accepted: 15 January 2020/Published online: 30 January 2020

(C) Canadian Anesthesiologists' Society 2020

Normal saline $(0.9 \% \mathrm{NaCl})$ is one of the most commonly administered maintenance and resuscitation fluids. If one considers the drug identification number on each bag, ${ }^{1,2}$ normal saline may be one of the most commonly administered drugs in medicine. Given the decades of experience with its use, low cost, and widespread availability, what could possibly be left to examine with respect to this medical staple?

In 1831, the first reports of intravenous fluid resuscitation of moribund patients were published as physicians aimed to replace lost cholera fluid with salt solutions balanced as closely as possible to that of serum. ${ }^{3,4}$ The term "normal saline" may have been originally colloquially applied to multiple isotonic salt solutions, despite many of these the solutions possessing very "nonnormal" physiologic parameters. ${ }^{3,4}$ The distinct "nonnormality" of $0.9 \% \mathrm{NaCl}$, the solution most referenced by the term normal saline, is further emphasized by the broad grouping of crystalloids into two major categories-normal saline and balanced salt solutions (e.g., Ringer's lactate and Plasma-Lyte). ${ }^{5}$

Infusions of normal saline produce a well-known hyperchloremic acidosis, while balanced salt solutions include buffers and have a decreased chloride content. ${ }^{2,5}$ Generally, the amount of normal saline infused as well as the degree of hyperchloremia reported seem to be associated with acidosis. ${ }^{5,6}$ The mechanism for this effect is uncertain. First, chloride is a significant anionic factor in proton $\left(\mathrm{H}^{+}\right)$balance, and hyperchloremia decreases the strong ion difference $(\mathrm{Na}+\mathrm{K}-\mathrm{Cl})$, which is a major

\footnotetext{
S. A. McCluskey, MD, PhD (ه) · J. Bartoszko, MD

Department of Anesthesia, University of Toronto, Toronto, ON, Canada

e-mail: Stuart.McCluskey@uhn.ca
}

determinant of $\mathrm{H}^{+}$concentration. A decrease in the strong ion difference is associated with metabolic acidosis, and, correspondingly, an increase in the strong ion difference is associated with metabolic alkalosis. ${ }^{4}$ Second, administration of a buffer-free crystalloid may dilute endogenous physiologic buffers, such as serum bicarbonate, thereby leading to a "dilutional acidosis". 2 Lastly, the restoration of effective circulating volume may contribute to acidosis in phases of clinical improvement, as previously hypo-perfused organs become better perfused. ${ }^{2}$ Additionally, chloride has complex effects pertaining to renal blood flow and glomerular filtration rate. Exogenous chloride may induce renal-specific vasoconstriction and reduce glomerular filtration rate. ${ }^{3,6,7}$ To complicate matters further, worsening hyperchloremia may also occur in the perioperative period independently of normal saline administration, such as in the setting of water loss in excess of chloride loss, as may occur with fever or fasting. ${ }^{8}$ These events may themselves be associated with decreased glomerular filtration rate.

While the metabolic derangements associated with normal saline are well documented, their association with patient outcomes is an active area of research. This includes perioperative patients undergoing major surgery, as the metabolic response to injury prominently includes an impaired ability to excrete excess salt and fluid loads. ${ }^{2,4}$ Acute postoperative hyperchloremia (serum chloride $>110$ $\mathrm{mEq} \cdot \mathrm{L}^{-1}$ ) had an incidence of $22 \%$ in a large retrospective observational study of 22, 851 diverse surgical patients. ${ }^{5}$ In this same study, hyperchloremic patients were at increased risk of 30-day mortality, postoperative renal dysfunction, and prolonged hospital length of stay. ${ }^{5}$

The association of hyperchloremia and normal saline administration with adverse renal outcomes and mortality is therefore highly relevant for perioperative kidney 
transplant recipients, particularly deceased-donor recipients. In these patients, a higher incidence of delayed graft function (DGF) is associated with poorer short and long-term outcomes. ${ }^{2,9}$ In this issue of the Journal, Nesseler et al. ${ }^{10}$ present a retrospective observational study of 359 adult deceased-donor kidney transplant recipients from a single institution. The study authors examined the association of postoperative hyperchloremia, hyperchloremic metabolic acidosis, an abbreviated strong ion difference, and volume of normal saline infused with patient outcomes. The primary outcome of interest, DGF, was defined as any need for renalreplacement therapy within one week of transplantation. A secondary sensitivity outcome included a decreased creatinine reduction ratio, defined as a fall in the ratio of postoperative creatinine of less than $30 \%$.

In the immediate postoperative $24 \mathrm{hr}, 11 \%(n=38)$ of patients developed hyperchloremia, 11\% $(n=38)$ developed hyperchloremic acidosis, 91\% $(n=328)$ developed a significant alteration in the abbreviated strong ion difference, and 20\% ( $n=70)$ experienced DGF. Using multivariable logistic regression, the authors found that normal saline infusion volume, but not hyperchloremia or hyperchloremic acidosis, was associated with an increased risk of DGF (odds ratio [OR], 1.14; 95\% confidence interval [CI], 1.00 to $1.29 ; P=$ 0.04 , for both logistic regression models including hyperchloremic acidosis and hyperchloremia).

The present study by Nesseler et al. ${ }^{10}$ is one of the larger perioperative studies published on this subject, however it may be limited by insufficient power to detect a clinically important difference. Patients who developed hyperchloremia or hyperchloremic acidosis received an average of approximately $1 \mathrm{~L}$ more normal saline than those who did not, with $P$ values that trended towards significance in this small cohort. There may be more to this data that is worth exploring. In this same vein, the CIs for the association of hyperchloremia and hyperchloremic acidosis with DGF did not exclude potential clinically important effects.

Despite these caveats, notable strengths of this study include the chosen endpoint of DGF, which is clinically relevant and of concern to both patients and providers. In addition, the single-centre nature of this study, while often traditionally cited as a disadvantage with respect to generalizability, may have helped reduce practice variability as a potential confounding factor, since the single institution from which patient data were collected appears to have had a reasonably uniform treatment approach to kidney transplant recipients, including indications for additional fluid administration. The use of goal-directed fluid therapy may be more appropriate as a basis for a multicentre trial, as there is tremendous variability in fluid management from one centre to another. Nevertheless, the effect of hyperchloremia on patient outcomes is likely to have an ever-diminishing effect as the risk of fluid excess is limited by improved clinical care and avoidance of unsubstantiated fluid protocols.

There are relevant clinical implications from large randomized-controlled trials in other patient populations. The SALT-ED trial was a multiple-crossover trial of 13,347 non-critically ill patients cared for outside an intensive care unit setting. While there was no difference in hospital-free days between the group that received balanced crystalloid and the group that received normal saline, the balanced crystalloid group had a lower incidence of major adverse kidney events than the saline group (adjusted OR, $0.82 ; 95 \% \mathrm{CI}, 0.70$ to $0.95 ; P=$ $0.01) .{ }^{11}$ The SMART trial included 15,802 critically ill adult patients treated in an intensive care environment, of which those randomized to the balanced crystalloid group had a lower rate of in-hospital mortality, new renalreplacement therapy, and persistent renal dysfunction. ${ }^{12}$ Notably, the absolute risk differences in these large trials were quite small, suggesting that the absolute risk difference attributable to normal saline in specific subpopulations may also be small. The near-ubiquitous administration of normal saline can make even small risk differences important at the population level. Finally, even the well-intentioned exclusive use of balanced salt solutions may not overcome the ill effects of either fluid excess or fluid restriction.

There is mounting evidence that the use of balanced salt solutions such as Ringer's lactate does not contribute to hyperkalemia in the renal transplant population, and may actually be safer than the use of normal saline. ${ }^{13}$ Indeed, one trial of adults undergoing kidney transplantation randomized to normal saline or Ringer's lactate intraoperatively was terminated early for safety after interim analysis revealed a higher rate of hyperkalemia requiring treatment in the normal saline group. ${ }^{14}$

The continued use of normal saline as a default first-line fluid for many practitioners deserves examination and debate. Balanced salt solutions are often as easy to access as normal saline, and, when indicated, appear to have few disadvantages. Had medicinal chemistry been able to make available today's balanced salt solutions during the pandemics of the 1800 's, it seems unlikely that "normal saline" would have emerged as the dominant colloquialism, where it appears to historically remain. 


\section{Chlorure et solution normal salin, ou le cheval et la charrue : l'association entre le choix de la solution cristalloïde et l'équilibre acide-base et les devenirs des patients récipiendaires de greffe rénale}

La solution normal salin $(\mathrm{NaCl}$ 0,9\%) constitue l'un des liquides de maintien et de réanimation les plus fréquemment administrés. Si l'on tient compte du numéro d'identification du médicament sur chaque sac, ${ }^{1,2}$ la solution normal salin pourrait être l'un des médicaments les plus fréquemment administrés en médecine. Étant donné les décennies d'expérience que nous possédons avec son emploi, son faible coût et sa grande disponibilité, que reste-t-il véritablement à explorer en ce qui touche à ce produit médical de base?

En 1831 étaient publiés les premiers comptes rendus de réanimation de patients moribonds grâce à des liquides intraveineux, alors que les médecins tentaient de remplacer les liquides perdus lors du choléra par des solutions salées dont l'équilibre imitait au mieux celui du sérum. ${ }^{3,4}$ Le terme de «solution normal salin » pourrait avoir été appliqué, à l'origine, à plusieurs solutions isotoniques salées, malgré le fait que bon nombre de ces solutions possédassent des paramètres physiologiques «non normaux ». ${ }^{3,4} \mathrm{La}$ 《non-normalité » spécifique du $\mathrm{NaCl}$ $0,9 \%$, la solution à laquelle il est le plus souvent fait référence par le terme de solution normal salin, est encore plus soulignée par le regroupement approximatif des cristalloïdes en deux grandes catégories - les solutions physiologiques salées, ou normal salin, et les solutions salines équilibrées (par ex., le lactate Ringer ou le PlasmaLyte). ${ }^{5}$

Les perfusions de solution normal salin produisent une acidose hyperchlorémique bien connue, alors que les solutions salines équilibrées contiennent des tampons et possèdent un contenu de chlorure diminué. ${ }^{2,5}$ En règle générale, la quantité de solution normal salin perfusée ainsi que le degré d'hyperchlorémie rapportée semblent être associés à l'acidose. ${ }^{5,6}$ Le mécanisme produisant cet effet est incertain. Premièrement, le chlorure est un important facteur anionique dans l'équilibre du proton $\left(\mathrm{H}^{+}\right)$, et l'hyperchlorémie diminue la différence d'ions forts $(\mathrm{Na}+$ $\mathrm{K}-\mathrm{Cl}$ ), un déterminant majeur de concentration $\mathrm{H}^{+}$. Une diminution de cette différence d'ions forts est associée à l'acidose métabolique; de la même façon, une augmentation de la différence d'ions forts est associée à une alcalose métabolique. 4 Deuxièmement, l'administration d'un cristallö̈de exempt de tampon pourrait diluer les tampons physiologiques endogènes tels que le bicarbonate sérique, entraînant ainsi une «acidose par dilution $»^{2}$ Enfin, la restauration du volume circulant efficace pourrait contribuer à l'acidose durant les phases d'amélioration clinique, au fur et à mesure que les organes précédemment hypoperfusés redeviennent mieux perfusés. ${ }^{2}$ En outre, le chlorure possède des effets complexes en ce qui touche au débit sanguin rénal et au taux de filtration glomérulaire. Le chlorure exogène pourrait induire une vasoconstriction spécifique au rein et réduire le taux de filtration glomérulaire. ${ }^{3,6,7}$ Pour compliquer la situation encore plus, une hyperchlorémie en augmentation pourrait également survenir en période périopératoire indépendamment de l'administration de solution normal salin, comme par exemple lors d'une perte d'eau plus importante que la perte de chlorure, comme cela peut survenir en cas de fièvre ou de jeûne. ${ }^{8}$ Ces événements pourraient en soi être associés à une réduction du taux de filtration glomérulaire.

Alors que les troubles métaboliques associés à une solution normal salin sont bien documentés, leur association aux devenirs des patients constitue un domaine de recherche actif. Ces recherches englobent les patients périopératoires subissant une chirurgie majeure, étant donné que la réponse métabolique aux lésions inclut de façon proéminente une capacité amoindrie d'éliminer les charges salines et liquidiennes excessives. ${ }^{2,4}$ Dans une vaste étude observationnelle rétrospective portant sur 22851 patients chirurgicaux, l'incidence d'hyperchlorémie postopératoire aiguë (chlorure sérique $>110 \mathrm{mEq} \cdot \mathrm{L}^{-1}$ ) était de $22 \% .{ }^{5}$ Dans cette même étude, les patients en hyperchlorémie couraient un risque accru de mortalité à 30 jours, de dysfonction rénale postopératoire, et de prolongation de la durée de séjour hospitalier. ${ }^{5}$

L'association d'une hyperchlorémie et d'une administration de solution normal salin à des complications rénales et de la mortalité est donc très pertinente pour les patients périopératoires recevant une greffe rénale, particulièrement pour les récipiendaires de donneurs décédés. Chez ces patients, une incidence plus élevée de retard de fonctionnement du greffon (RFG) est associée à de moins bons devenirs, tant à court qu'à long terme. ${ }^{2,9}$ Dans ce numéro du Journal, Nesseler et coll. ${ }^{10}$ présentent une étude observationnelle rétrospective monocentrique portant sur 359 adultes récipiendaires de greffe rénale de donneurs décédés. Les auteurs de l'étude ont examiné l'association entre l'hyperchlorémie postopératoire, l'acidose métabolique hyperchlorémique, la différence d'ions forts abrégée, le volume de solution normal salin perfusé et les devenirs des patients. Le critère 
d'évaluation principal, soit le RFG, était défini comme tout besoin de traitement de suppléance rénale dans la première semaine suivant la greffe. Un critère d'évaluation secondaire de sensibilité incluait un rapport de réduction de la créatinine plus bas, défini comme une diminution dans le rapport de créatinine postopératoire de moins de 30 $\%$.

Dans les 24 heures postopératoires immédiates, $11 \%$ ( $n$ = 38) des patients ont manifesté une hyperchlorémie, $11 \%$ $(n=38)$ une acidose hyperchlorémique, $91 \%(n=328)$ une altération significative dans la différence d'ions forts abrégée, et $20 \%(n=70)$ ont subi un RFG. À l'aide d'une analyse de régression logistique multivariée, les auteurs ont découvert que le volume de perfusion de solution normal salin, et non l'hyperchlorémie ou l'acidose hyperchlorémique, était associé à un risque accru de RFG (rapport de cotes [RC], 1,14; intervalle de confiance [IC] $95 \%, 1,00$ à 1,$29 ; P=0,04$ pour les deux modèles de régression logistique comprenant l'acidose hyperchlorémique et l'hyperchlorémie).

L'étude de Nesseler et coll. ${ }^{10}$ présentée ici est l'une des plus vastes études périopératoires publiées à ce sujet, mais elle pourrait tout de même être limitée par un certain manque de puissance pour détecter une différence significative d'un point de vue clinique. Les patients ayant développé une hyperchlorémie ou une acidose hyperchlorémique ont reçu en moyenne 1 L supplémentaire de solution normal salinpar rapport à ceux n'ayant pas souffert de ces complications, avec des valeurs de $P$ tendant vers la signification clinique dans cette petite cohorte. Ces données pourraient contenir d'autres informations dignes d'être explorées. Dans la même veine, les intervalles de confiance portant sur l'association de l'hyperchlorémie et de l'acidose hyperchlorémique au RFG n'excluaient pas d'effets potentiellement importants d'un point de vue clinique.

Malgré ces réserves, les forces de cette étude comprennent le critère retenu de RFG, qui est pertinent d'un point de vue clinique et intéressant tant pour les patients que pour les fournisseurs de soins. En outre, la nature monocentrique de cette étude, bien que souvent citée comme désavantage en ce qui touche à l'aspect généralisable des résultats d'étude, pourrait ici avoir au contraire favorisé la réduction de la variabilité de pratique comme facteur confondant potentiel, étant donné que le centre unique duquel les données des patients ont été colligées semble disposer d'une approche raisonnablement uniforme du traitement des récipiendaires de greffe rénale, y compris en ce qui a trait aux indications concernant l'administration de liquide supplémentaire. L'utilisation d'une thérapie liquidienne orientée vers un but pourrait être plus appropriée comme fondement pour une étude multicentrique, étant donné qu'il existe une importante variabilité dans la prise en charge liquidienne d'un centre à un autre. Toutefois, l'effet de l'hyperchlorémie sur les devenirs des patients aura probablement un effet de plus en plus réduit au fur et à mesure que le risque d'excès liquidien s'amoindrit grâce à l'amélioration des soins cliniques et à l'abandon des protocoles liquidiens non fondés.

Il existe des implications cliniques pertinentes d'études randomisées contrôlées d'envergure réalisées auprès d'autres populations de patients. L'étude SALT-ED était un essai croisé multiple réalisé chez 13347 patients en état non critique et pris en charge hors d'un contexte de soins intensifs. Alors qu'aucune différence n'a été observée en matière de jours sans hospitalisation entre le groupe ayant reçu un cristalloïde équilibré et le groupe ayant reçu une solution normal salin, le groupe cristalloïde équilibré a démontré une incidence plus faible de complications rénales majeures que le groupe solution normal salin (RC ajusté, 0,82 ; IC $95 \%, 0,70$ à 0,$95 ; P=0,01) .{ }^{11}$ L'étude SMART incluait quant à elle 15802 patients adultes en état critique et traités dans un environnement de soins intensifs, et les patients randomisés à recevoir le cristalloïde équilibré ont affiché un taux plus faible de mortalité hospitalière, de nouveau traitement de suppléance rénale, et de dysfonctionnement rénal persistant. ${ }^{12}$ Fait à noter, les différences de risque absolu dans ces grandes études étaient plutôt faibles, ce qui laisse penser que la différence de risque absolu attribuable à la solution normal salin pourrait également être faible chez certaines souspopulations spécifiques. L'administration quasiomniprésente de solution normal salin peut rendre même de petites différences de risque importantes au niveau d'une population. Enfin, même l'utilisation exclusive et bien intentionnée de solutions salines équilibrées pourrait ne pas suffire à vaincre les effets délétères d'un excès ou au contraire d'une restriction de liquides.

Les données probantes s'accumulent concernant le fait que l'utilisation de solutions salines équilibrées telles que le lactate Ringer ne contribue pas à l'hyperkaliémie dans une population de greffés rénaux, et pourrait en fait être plus sécuritaire que l'utilisation d'une solution normal salin. ${ }^{13}$ En effet, une étude portant sur des adultes subissant une greffe rénale et randomisés à recevoir de la solution normal salin ou du lactate Ringer en période peropératoire a été précocement interrompue pour des raisons de sécurité après qu'une analyse intérimaire a révélé un taux plus élevé d'hyperkaliémie nécessitant un traitement dans le groupe recevant une solution normal salin. ${ }^{14}$

L'utilisation continue de solution normal salin comme liquide de première ligne par défaut par de nombreux praticiens mérite d'être examinée et débattue. Les solutions salines équilibrées sont souvent aussi accessibles que la solution normal salin et, lorsqu'indiquées, elles semblent 
n'avoir que de rares désavantages. Si la chimie médicinale avait pu rendre disponibles les solutions salines équilibrées d'aujourd'hui pendant les épidémies du XIXe siècle, il semble peu probable que le terme de «solution normal salin » ait été retenu comme l'expression familière dominante qu'elle est aujourd'hui, où elle semble demeurer historiquement.

Conflicts of interest None.

Funding statement None.

Editorial responsibility This submission was handled by Dr. Philip M. Jones, Associate Editor, Canadian Journal of Anesthesia.

\section{Conflit d'intérêt Aucun.}

Déclaration de financement Aucune.

Responsabilité éditoriale Cet article a été traité par Dr Philip M. Jones, rédacteur adjoint, Journal canadien d'anesthésie.

\section{References}

1. Severs D, Rookmaaker MB, Hoorn EJ. Intravenous solutions in the care of patients with volume depletion and electrolyte abnormalities. Am J Kidney Dis 2015; 66: 147-53.

2. Hoorn EJ. Intravenous fluids: balancing solutions. J Nephrol 2017; 30: 485-92.

3. Lobo DN, Awad S. Should chloride-rich crystalloids remain the mainstay of fluid resuscitation to prevent 'pre-renal' acute kidney injury?: con. Kidney Int 2014; 86: 1096-105.

4. Awad S, Allison SP, Lobo DN. The history of $0.9 \%$ saline. Clin Nutr 2008; 27: 179-88.
5. McCluskey SA, Karkouti K, Wijeysundera D, Minkovich L, Tait $G$, Beattie WS. Hyperchloremia after noncardiac surgery is independently associated with increased morbidity and mortality: a propensity-matched cohort study. Anesth Analg 2013; 117: 412-21.

6. Waters JH, Miller LR, Clack S, Kim JV. Cause of metabolic acidosis in prolonged surgery. Crit Care Med 1999; 27: 2142-6.

7. Chowdhury AH, Cox EF, Francis ST, Lobo DN. A randomized, controlled, double-blind crossover study on the effects of 2-L infusions of $0.9 \%$ saline and Plasma-Lyte ${ }^{\circledR} 148$ on renal blood flow velocity and renal cortical tissue perfusion in healthy volunteers. Ann Surg 2012; 256: 18-24.

8. Nagami GT. Hyperchloremia - why and how. Nefrología 2016; 36: $347-53$.

9. Mannon RB. Delayed graft function: the AKI of kidney transplantation. Nephron 2018; 140: 94-8.

10. Nesseler N, Rached A, Ross JT, et al. Association between perioperative normal saline and delayed graft function in deceased-donor kidney transplantation: a retrospective observational study. Can J Anesth 2020; 67: DOI: https://doi. org/10.1007/s12630-020-01577-9.

11. Self WH, Semler $M W$, Wanderer JP, et al. Balanced crystalloids versus saline in noncritically ill adults. N Engl J Med 2018; 378: 819-28.

12. Semler $M W$, Self $W H$, Wanderer JP, et al. Balanced crystalloids versus saline in critically ill adults. N Engl J Med 2018; 378: 82939.

13. Orbegozo Cortés D, Rayo Bonor A, Vincent JL. Isotonic crystalloid solutions: a structured review of the literature. $\mathrm{Br} \mathrm{J}$ Anaesth 2014; 112: 968-81.

14. O'Malley CM, Frumento RJ, Hardy MA, et al. A randomized, double-blind comparison of lactated Ringer's solution and $0.9 \%$ $\mathrm{NaCl}$ during renal transplantation. Anesth Analg 2005; 100: 1518-24.

Publisher's Note Springer Nature remains neutral with regard to jurisdictional claims in published maps and institutional affiliations. 